\title{
Design, fabrication and perivascular implantation of bioactive scaffolds engineered with human adventitial progenitor cells for stimulation of arteriogenesis in peripheral ischemia
}

M Carrabba ${ }^{1,+}$, C De Maria ${ }^{1,+}$, A Oikawa ${ }^{2}$, C Reni $^{2}$, I Rodriguez-Arabaolaza ${ }^{2}$, H Spencer $^{2}$, S Slater $^{2}$, E Avolio $^{2}$, Z Dang $^{2}$, G Spinetti $^{3}$, P Madeddu $^{2}$ and G Vozzi ${ }^{1}$

${ }^{1}$ Research Centre 'E. Piaggio', University of Pisa, Italy

${ }^{2}$ Bristol Heart Institute, School of Clinical Sciences, University of Bristol, Bristol, UK

${ }^{3}$ IRCCS Multi Medica, Milan, Italy

${ }^{+}$These authors contributed equally to this work

Address of corresponding authors

Giovanni Vozzi, Research Centre 'E. Piaggio',University of Pisa, Largo Lucio Lazzarino 2, 56126 Pisa, Italy. E-mail: g.vozzi@ing.unipi.it

Paolo Madeddu, Bristol Heart Institute, University of Bristol, Upper Maudlin Road, Bristol BS2 8HW, United Kingdom, Email: Paolo.Madeddu@bristol.ac.uk 
Abstract - Cell therapy represents a promising option for revascularization of ischemic tissues. However, injection of dispersed cells is not optimal to ensure precise homing into the recipient's vasculature. Implantation of cell-engineered scaffolds around the occluded artery may obviate these limitations. Here, we employed the synthetic polymer polycaprolactone (PCL) for fabrication of 3D woodpile- or channel-shaped scaffolds by a computer-assisted writing system (Pressure Assisted Micro-syringe Square $\left(\mathrm{PAM}^{2}\right)$ ), followed by deposition of gelatin (GL) nanofibers by electrospinning. Scaffolds were then cross-linked with natural (genipin, GP) or synthetic (3-glycidyloxypropyl-trimethoxy-silane, GPTMS) agents to improve mechanical properties and durability in vivo. The composite scaffolds were next fixed by crown inserts in each well of a multi-well plate and seeded with adventitial progenitor cells (APCs, 3 cell lines in duplicate), which were isolated/expanded from human saphenous vein surgical leftovers. Cell density, alignment, proliferation and viability were assessed 1 week later. Data from in vitro assays showed channelshaped/GPTMS-crosslinked scaffolds confer APCs with best alignment and survival/growth characteristics. Based on these results, channel-shaped/GPTMS-crosslinked scaffolds with or without APCs were implanted around the femoral artery of mice with unilateral limb ischemia. Perivascular implantation of scaffolds accelerated limb blood flow recovery, as assessed by laser Doppler or fluorescent microspheres, and increased arterial collaterals around the femoral artery and in limb muscles compared with non-implanted controls. Blood flow recovery and perivascular arteriogenesis were additionally incremented by APC-engineered scaffolds. In conclusion, perivascular application of human APC-engineered scaffolds may represent a novel option for targeted delivery of therapeutic cells in patients with critical limb ischemia. 


\section{Introduction}

Peripheral artery disease (PAD) is a common pathological condition that affects up to $15 \%$ of people older than 55 years [1]. Critical limb ischemia (CLI), the most severe manifestation of PAD, causes poor quality of life and requires foot amputation in $25 \%$ of cases within one year from the diagnosis. The reported amputation and mortality rates exceed $50 \%$ in CLI patients that are unsuitable for surgical or endovascular revascularization [2]. Considering these unfavorable outcomes, new therapeutic approaches are urgently needed.

Stem cell therapy has been recently introduced as a novel approach for treatment of CLI patients [3-5]. Clinical trials using bone marrow (BM)-derived cells showed initial evidence of safety and therapeutic efficacy [6-9]. However, current delivery methodologies are not efficient enough to achieve optimal distribution of the cell product. The intra-arterial route is unsuitable for patients with multiple occlusions, as cells will not reach the ischemic tissue by blood flow. Intra-muscular delivery of dispersed cells results in stimulation of patchy microvascular angiogenesis below the occlusion site, which may be insufficient to promote an efficient reperfusion. An innovative method consists of transplanting stem cells perivascularly, e.g. along the blocked limb arteries, with the intention to increase the formation of arterial collaterals bypassing the obstruction. In addition, delivery of stem cells embedded in scaffolds or microparticles has been proposed for improving cell viability, tolerogenicity and paracrine activity [10-13]. We have recently documented the advantages of combining the two approaches. In a mouse model of limb ischemia, perivascular delivery of microbeads filled with human mesenchymal stem cells (MSCs) stimulated the growth of arterioles alongside the occluded femoral artery, thereby improving perfusion, oxygenation and limb salvage [14]. The tributary microvascular bed was also enhanced, as documented by increased counts of capillaries and arterioles in the ischemic adductor. Therefore, the method allows for additive implementation of proximal collateralization and distal microvascular angiogenesis. However, cell delivery by microbeads has also limitations. First, microencapsulated cells are unavailable for direct participation in vascular reparative remodelling. Second, random distribution in the target tissue does not ensure the development of an ordered neovascularization.

To circumvent these problems, here we propose novel bio-engineered scaffolds providing a temporary artificial extracellular matrix $(\mathrm{ECM})$ to support cell engraftment and guide the formation of a well-aligned arterial collateralization [15, 16]. The present study comprises four main workpackages: (1) Fabrication of 3D scaffolds, (2) Functionalization of scaffolds with human adventitial progenitor cells (APCs), (3) In vitro testing of cellularized scaffolds, and (4) In vivo perivascular transplantation of cellularized scaffolds in a mouse model of limb ischemia.

Woodpile- or channel-shaped polycaprolactone (PCL) scaffolds were fabricated using a computer-assisted writing approach (Pressure Assisted Micro-syringe Square (PAM ${ }^{2}$ ) [17]), which allows to precisely dictating the internal architecture of a 3D structure at the microscale level, thus facilitating greater control of cell engineering. This step was followed by deposition of gelatin (GL) 
nanos by electrospinning. The system uses a high voltage field to launch a jet of GL strand onto the scaffold, thereby forming a nanofibre network with adhesive features similar to those of the natural ECM [18]. Two crosslinking agents, $\gamma$-glycidoxypropyltrimethoxysilane (GPTMS) and Genipin (GP), were used to increase scaffold durability. As a cellular source for scaffold functionalization, we used APCs isolated/expanded from remnants of saphenous vein of coronary artery bypass graft surgery. APCs are attracting considerable attention for applications of cardiovascular regenerative medicine and have been developed to a clinical-grade cell product in our laboratory [19, 20]. In models of limb and myocardial ischemia, transplantation of APCs promotes vascularization through the release of angiocrine factors and chemokines that recruit endothelial cells, pro-angiogenic mononuclear cells, and resident progenitor cells [21-24]. Following successful seeding of APCs, the functionalized scaffolds were tested in in vitro assays and then implanted around the femoral artery of mice undergoing operative limb ischemia. Results of in vivo studies indicate the feasibility and therapeutic efficacy of the proposed approach.

\section{Materials and methods}

\subsection{Ethics}

Studies complied with the principles stated in the "Declaration of Helsinki" and were covered by approval (06/Q2001/197) from the Bath Research Ethics Committee. Patients gave written informed consent to be recruited in the study. Experiments involving live animals were performed in accordance with the Guide for the Care and Use of Laboratory Animals (the Institute of Laboratory Animal Resources, 1996) and with approval of the British Home Office and the University of Bristol.

\section{2. $P A M^{2}$ fabrication}

$\mathrm{PAM}^{2}$ is a modular microfabrication system able to dispose high and low viscosity solutions following precise spatial patterns. It is based on a 3axis micropositioner for the movement in space of different fabrication tools ( 1 axis, defined as $\mathrm{z}$ ) respect to a working plane ( 2 axes, defined as $\mathrm{x}-\mathrm{y}$ plane) under the control of a dedicated CAD/CAM software. In the present work the air-pressure extrusion system was used, composed by a stainless steel syringe with a glass capillary needle of $80 \mu \mathrm{m}$ diameter $[25,26]$. Polycaprolactone (PCL), from Sigma Aldrich, was solved in chloroform (Sigma Aldrich) $10 \% \mathrm{w} / \mathrm{v}$, loaded into the syringe and extruded. Immediately after the extrusion the chloroform evaporates, allowing the shape retention. The 3D structures are obtained following a layer-by-layer approach, using hydrofilm as support material. Two different geometries were designed:

1. channels $150-200 \mu \mathrm{m}$ wide and $70 \mu \mathrm{m}$ high arranged in $100 \mu \mathrm{m}$-wide lines. The bottom part of the channel was composed by a grid layer, built also with the $\mathrm{PAM}^{2}$ technique, with the aim at avoiding that the channel structures collapse at the time of detachment from the deposition plate; 
2. woodpiles composed by 6 layers of PCL interconnected pores with size of $200 \mu \mathrm{m}$ per side and $100 \mu \mathrm{m}$-wide lines.

The fabrication process was finely calibrated: the working parameters were the deposition plane velocity of $9 \mathrm{~mm} / \mathrm{s}$ and extrusion pressure of $8 \mathrm{kPa}[25,26]$.

\subsection{Electrospinning fabrication}

The electrospinning process has been subjected to a tuning of parameters allowing the selection of the desired characteristics of the nanofibres and the repeatability of the experiments. Tests were performed using a solution of GL from porcine skin (Sigma Aldrich) dissolved in 60/40\% (v/v) acid acetic/distilled water at a concentration of $10 \%(\mathrm{w} / \mathrm{v})$; the solution was then stirred at $50^{\circ} \mathrm{C}$ for 60 min. While keeping the spinning time constant $(12 \mathrm{~min})$, the following working parameters of the electrospinning apparatus (Linari Engineering S.r.l., Italy) were varied to achieve the most performant fibres based of the diameter of section, porosity and percentage of defects of the electrospun material:

- $\quad$ Distance between tip and collector: 15, 20 and 30cm;

- $\quad$ Voltage: 30,40 and $50 \mathrm{kV}$;

- $\quad$ Feed/Flow rate: 1,2 and $3 \mathrm{ml} / \mathrm{hour}$;

The morphology of the samples was evaluated by scanning electron microscopy (SEM). Features and porosity of gelatin nanofibres were assessed by image processing (Image $\mathrm{J}$ ).

\subsection{Combination of $P A M^{2}$ and electrospinning}

The manufacturing of the final composite scaffold was carried by a two steps procedure: the microstructures, fabricated with the $\mathrm{PAM}^{2}$ system, were placed in the electrospinning device. The $3 \mathrm{D}$ channels and woodpiles were uniformly fasten on the collector and the fibers were deposited on top of the surface, forming a mat of uniform thickness of randomly oriented gelatin nanofibers. Considering the small size of the microstructures, their presence do not alter the optimal electrospinning parameters for obtaining a uniform and controlled gelatin mesh. Thus, on the basis of the results extensively described in paragraph 3.2, electrospinning parameters were set as follow: distance from the needle $20 \mathrm{~cm}$; voltage $50 \mathrm{kV}$; flow $1 \mathrm{ml} /$ hour; time of spinning $12 \mathrm{~min}$.

\subsection{Crosslinking Procedure}

Crosslinking treatments were performed to prevent rapid GL dissolution in water and preserve the peculiar biomimetic morphology. Two cross-linking systems were compared, using GPTMS (Sigma Aldrich, Milan, Italy) [27] and GP (Challenge Bioproducts Ltd., Taichung, Taiwan) [28]:

i. GL was dissolved in 60/40 v/v acid acetic/distilled water at a concentration of $10 \%(\mathrm{w} / \mathrm{v})$ at $50^{\circ} \mathrm{C}$. GPTMS cross-linked GL nanofibres were prepared adding $200 \mu \mathrm{l}$ of GPTMS per gram of GL and the solution was mixed $1 \mathrm{~h}$ before spinning. After spinning, samples were dried at $37^{\circ} \mathrm{C}$ for 24 hours.

ii. GL was dissolved in 60/40\% (v/v) acid acetic/distilled water at a concentration of $30 \%(\mathrm{w} / \mathrm{v})$ and stirred at $50^{\circ} \mathrm{C}$ for $60 \mathrm{~min}$. Then $60 \mathrm{mg}$ of GP dissolved in $0.5 \mathrm{ml}$ of ethanol and $1 \mathrm{ml}$ of 
phosphate-buffered saline (PBS) were added to $10 \mathrm{ml}$ of the GL solution. Thirty minutes later, the mixture was ready to be electrospun. To complete the crosslinking, samples were soaked in 5\% (w/v) GP in ethanol solution for 7 days at $37^{\circ} \mathrm{C}$. The mats were rinsed in $0.1 \mathrm{M}$ PBS, pH 7.4, dried overnight at $37^{\circ} \mathrm{C}$, then rinsed in ethanol and dried again.

\subsection{Mechanical characterization}

Mechanical properties of scaffolds were measured using Zwick/Roell Z005 device (Zwick GmbH \& Co, Germany). At least three specimens for each architecture (channels and woodpiles) were tested. The samples were rectangular shaped presenting a length of $9 \pm 1 \mathrm{~mm}$ (in the tensile direction), a width of $6 \mathrm{~mm} \pm 1 \mathrm{~mm}$ and a thickness of $70 \mu \mathrm{m} \pm 10 \mu \mathrm{m}$; measurements were performed using a micrometer. Samples were connected using a custom-made gripping system to the testing device and subjected to tensile tests with a strain rate of $0.01 \mathrm{~min}^{-1}$ until failure. The channel structures were tested both in axial (x) and longitudinal (y) direction. Stress-strain curves were derived from force-displacement data. In particular the stress was calculated dividing the force by the apparent area given by the product of the width and the thickness, not taking in consideration the internal geometry of the scaffolds. The elastic modulus of each sample was evaluated from the slope of the first linear portion of the stress-strain curve.

\subsection{Cell isolation and culture}

APCs were obtained from vein leftovers using a standard operating protocol described previously [21]. In brief, saphenous veins were carefully dissected from surrounding tissues using a sterile scalpel and then thoroughly washed in PBS. Veins were manually minced prior to 4 hour incubation with $3.7 \mathrm{mg} / \mathrm{mL}$ Liberase 2 (Roche Technologies). Single cell suspension was ensured by passing the dissociated material through a $30 \mu \mathrm{m}$ cell strainer. The suspension was then depleted of endothelial cells using anti-CD31 conjugated beads (Miltenyi Biotech), followed by positive selection for CD34+ cells by anti-CD34 beads (Miltenyi Biotech). The immunosorted CD34+ cells were then plated on fibronectin $(10 \mu \mathrm{g} / \mathrm{mL})$ coated plates in presence of EGM2 medium (Lonza) supplemented with $2 \%$ FBS. Adherent colonies were passaged to new culture dishes once they reached 60-70\% confluence. All in vitro experiments were set up with cells at P6. Purity of the preparations was determined by flow cytometry using combinations of the following antibodies to confirm typical phenotype: antiCD44 (ebioscience), anti-CD-105 (Life Technologies) and anti-CD90 (BD biosciences). After staining, fluorescence was analyzed using a FACS Canto II flow cytometer and FACS Diva software (both BD Biosciences). Flow-cytometry analysis indicates that $>95 \%$ cells express the surface antigens CD105, CD44 and CD90.

\subsection{Cell seeding and in vitro assays}

Scaffolds were cut in pieces of 6x6 mm per side, immobilized in 48 well-plates (CELLSTAR ${ }^{\circ}$ ) using CellCrown (Sigma Aldrich) and sterilized with a wash of Ethanol/Water $70 \%$ (v/v) and UV exposition of 15 min on each side. Three APC lines were seeded onto scaffolds at the concentration of 10,000 cells/well and tested as duplicates in the following in vitro assays. 


\subsubsection{Cell counting}

We first compared the ability of APCs to grow as adherent monolayers onto different scaffolds. To facilitate recognition and counting, cells were stained with the fluorescent marker 1,1'-Dioctadecyl3,3,3',3'-Tetramethylindocarbocyanine Perchlorate (Dil) (CellTrackerTM CM-DiI, Molecular Probes, Leiden, Netherlands). Histological assessment indicates that the efficiency of the marking is $>95 \%$. After 7 days of incubation, the culture medium was removed and scaffolds with attached cells were rinsed with PBS and fixed with paraformaldehyde solution (PFA, Sigma-Aldrich). Nuclei were stained with 4, 6-diamidino-2-phenylindole (DAPI). Samples were analyzed under Fluorescence Microscope and images were analyzed using ImageJ to determine cell density.

\subsubsection{Viability assay}

APC viability was evaluated using the MTS assay (MTS CellTiter $96^{\circledR} \mathrm{AQ}_{\text {ueous }}$ Non-Radioactive Cell Proliferation Assay, Promega Corporation).

\subsubsection{Proliferation assay}

Ability of cells to proliferate once seeded onto scaffolds was evaluated by Click-iT® EdU Assay (Life Technologies Corporation). Nucleoside 5-ethynyl-2'-deoxyuridine (EdU) is incorporated into DNA during cell replication.

\subsubsection{Differentiation assay}

The ability of APCs to differentiate into endothelial cells (ECs) or vascular smooth muscle cells (VSMCs) was tested following exposure to inductive media [21]. Cells were seeded either onto glass coverslips or onto scaffolds at a density of 9000 cells $/ \mathrm{cm}^{2}$. After 48 hours, EGM-2 medium was substituted with differentiation media, with subsequent changes every 3 days, for 14 days. The following differentiation protocols were used: (1) EC differentiation: cells were cultured with CFUHill Liquid Medium Kit (StemCell Technologies, UK); (2) VSMC differentiation: cells were cultured in basal medium (60\% DMEM low glucose $+40 \%$ MCDB201) added with $20 \mathrm{ng} / \mathrm{mL}$ human PDGFBB (Pepro-Tech). As a control, APCs cultured in EGM-2 were included. For immunofluorescence staining, cells were fixed with freshly prepared $4 \%(\mathrm{w} / \mathrm{v})$ paraformaldehyde for 20 minutes at room temperature (RT), washed with PBS and permeabilized with 0.1\% (v/v) Triton X100 (Sigma-Aldrich) diluted in PBS for 10 minutes at RT when detection of intracellular antigens was required. The following antibodies were used: (1) for endothelial cell characterization: VE-Cadherin (1:50, Santa Cruz), von Willebrand Factor (1:50, DAKO, UK) and CD31 (1:50); (2) VSMC characterization: $\alpha-$ smooth-muscle actin (1:100, DAKO), $\alpha$-retinol-binding protein 1 (1:100, Abcam), and $\alpha$-calponin (1:100, Abcam). Primary antibodies were incubated for 16 hours at $4^{\circ} \mathrm{C}$. Secondary antibodies were incubated for 1 hour at $20^{\circ} \mathrm{C}$ in the dark (1:200 goat anti-rabbit Alexa 488, 1:200 goat anti-mouse Alexa 488 (Life Technologies, UK). The nuclei were counterstained with 40,6-diamidino-2phenylindole (DAPI) (Sigma-Aldrich). Coverslips and scaffolds were mounted using Fluoromount-G (Sigma-Aldrich). Cells were analyzed at X400 magnification. 


\subsection{In vivo tests}

The ability of APC-engineered scaffolds to promote formation of arterial collaterals and microvessels was investigated in a mouse model of unilateral limb ischemia [14]. CD1 mice underwent femoral artery occlusion by silk ligature and were randomly assigned to 3 experimental groups ( $n=6$ mice per group), given no treatment (control), implantation of scaffolds (properly dimensioned in $2 \times 2 \mathrm{~mm}$ squares) without cells, or implantation of scaffolds seeded with APCs. For this last step of the investigation, we used the channel-shaped scaffold with nanofibers crosslinked by GPTMS, which showed the best in vitro characteristics, as described in Results section. Hemodynamic recovery was followed up using laser Doppler flowmetry as previously reported. In addition, adductor muscle blood flow was measured by fluorescent microspheres [29].

Immunohistochemistry was used to count capillaries, arterioles and infiltrating cells in the area of implantation. At sacrifice, the adductor muscle and the area that includes the femoral artery and scaffold were explanted, fixed with paraformaldehyde (PFA) (4\% w/v in PBS) and then embedded in cryogenic medium. The embedded sample was fixed into a microtome at $-20^{\circ} \mathrm{C}$ and sectioned in 5-8 $\mu \mathrm{m}$ thick slices. Vascular structures were recognized using antibodies against $\alpha$-SMACy3 which identifies vascular smooth muscle cells (VSMCs) and Isolectin B4 Biotin Conjugate which stains ECs. Furthermore, infiltrating monocytes and macrophages were identified using antibodies against CD3- $\varepsilon$ (Hamster anti mouse) and F4/80 (Rat anti Mouse), respectively. Additionally, in separate experiments, the persistence of cells ( $n=2$ APCs lines, $n=4$ mice) at 3 weeks from implantation was studied by labelling them with Dil and then performing confocal microscopy analyses of implanted muscles.

\subsection{Statistical Analysis}

Experiments were performed using randomized/blinded protocols. Group identity was decoded only after the completion of analyses. Results are expressed as mean \pm standard error. An initial test was performed to determine the normal distribution of data and the applicability of parametric or nonparametric tests. Analysis of laser Doppler blood flow recovery was performed using repeated measures Two-Way ANOVA followed by pair-wise comparison using the Holm-Sidak method. In other experiments, study endpoints were analyzed using one-way ANOVA, followed by pair post-test comparison using Tukey test. When the normality test failed, differences between groups were analyzed using Kruskal-Wallis test. A p-value of $<0.05$ was considered statistically significant.

\section{Results}

\subsection{Analysis of PAM ${ }^{2}$ microstructures}

Using optical microscopy we first confirmed that fabrication of the woodpile and channels architecture was achieved with the desired features. The 3D structures have a thicknesses around 60- 
$75 \mu \mathrm{m}$. Woodpiles demonstrate to have holes of $200 \mu \mathrm{m}$, with a line of deposition between 100 and $120 \mu \mathrm{m}$. Additionally, channels were $200 \mu \mathrm{m}$ wide, with walls $100-120 \mu \mathrm{m}$ thick (figure 1 ).

\subsection{Characterization of crosslinked nanofibres}

The morphology, size and shape of the fibres deposited using different electrospinning parameters was verified by scanning electron microscopy (figure 2).

Results, illustrated in figure 3(A), indicate an inversely proportional dependence of the section diameter on the tip-collector distance $(300 \pm 20 \mathrm{~nm}$ at $15 \mathrm{~cm}, 230 \pm 1 \mathrm{~nm}$ at $20 \mathrm{~cm}$, and $200 \pm 10 \mathrm{~nm}$ at 30 $\mathrm{cm})$ and applied voltage $(215 \pm 15 \mathrm{~nm}$ at $30 \mathrm{kV}, 190 \pm 10 \mathrm{~nm}$ at $40 \mathrm{kV}$, and $165 \pm 8 \mathrm{~nm}$ at $50 \mathrm{kV})$. Analysis of the influence of feed rate showed that for low values of flow the diameter section increases from $207 \pm 7 \mathrm{~nm}$ at $1 \mathrm{ml} /$ hour to $225 \pm 10 \mathrm{~nm}$ at $2 \mathrm{ml} /$ hour, and $240 \pm 5 \mathrm{~nm}$ at $3 \mathrm{ml} /$ hour. The voltage level of $50 \mathrm{kV}$ showed the highest repeatability as assessed by the standard error values and therefore this parameter was kept constant in further evaluation of fibre porosity and defects, when varying flow and tip-collector distance.

The thin layer of nanofibres produced during the process (thickness lower than $10 \mu \mathrm{m}$ ) allowed the assessment of the area associated to pores by image analysis (Image J). Porosity was defined as the ratio between the number of pixels associated with pores and the total number of pixels:

$$
p=\left(\frac{n^{\circ} \text { pix }}{n^{\circ} \text { pix }}\right) \times 100
$$

Typical defects illustrated in figure 3(B) consist of irregularity due to formation of polymer beads or fusion of several fibre bundles leading to loss of morphological properties of the mat. SEM pictures were analyzed and defects were quantified by imaging processing (figure $3(\mathrm{C})$ ). They were calculated by the ratio between the number of pixels associated with the defects and the total number of pixels associated to the fibres:

$$
d f=\left(\frac{n^{\circ} \text { pix }}{n^{\circ} \text { pix }}\right) \times 100
$$

The percentage of the defects ( $d f$ ) for flow of $1 \mathrm{ml} /$ hour was significantly lower compared with higher flow rates. Both $1 \mathrm{ml} /$ hour and $3 \mathrm{ml} /$ hour showed high and appropriate levels of nanofibres porosity (around $80 \%$ ) (figure 4(A-B)). Porosity is among the most important features of scaffolds, providing cell communication, exchange of nutrients or waste elimination and increasing the surface areavolume ratio. This allows the presence of more sites for cell adhesion, spreading and bridging. Figure 4(D-E) shows that distances of 20 and $30 \mathrm{~cm}$ reduce significantly the percentage of defects (below $1 \%)$ and yield the best porosity features. 
In order to have an objective criterion for choosing the definitive working condition, the R1 parameter, defined as the ratio between the pixels associated to defects and pixels associated to fibres, was calculated (figure $4(C)$ ). The lowest R1 value was then chosen to denote the optimum flow rate. Likewise, the ratio between the defects and fibres was determined for different distances to calculate R2 (figure 4(F)). The following parameters were then fixed for the setup of the electrospinning process: voltage $50 \mathrm{kV}$, distance $20 \mathrm{~cm}$ and flow rate $1 \mathrm{ml} /$ hour. Figure $5(\mathrm{~A})$ shows the nanofibre obtained as results from tuning of the electrospinning system. From the image processing they have the average diameter section of $210 \pm 49 \mathrm{~nm}$. Layer of nanofibres fabricated with the chosen parameters showed a pore distribution ranging from $0.4 \mu \mathrm{m}$ to around $4 \mu \mathrm{m}$ of diameter (average: $0.95 \pm 0.04 \mu \mathrm{m}$, figure $5(\mathrm{~B})$ ). Previous studies have reported that such range of pore size and fibre section are the most suitable to achieve good cell viability and proliferation on porous matrices [27, 30-33].

The final step of multi-scale microfabrication consisted of merging the two techniques to incorporate GL nanofibres within the PCL microstructure. Dimensions of PCL lines and nanofibres, tuned previously, were confirmed with repeatability after the manufacturing steps (figure 5(C)).

\subsection{Mechanical characterization}

From the stress-strain curves, the elastic modulus, the ultimate stress and ultimate strain were calculated. The results show that different types of structures have different rigidity (figure 6(A)): the

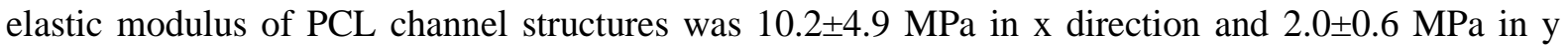
direction, while that of PCL woodpile structures was 4.2 $\pm 0.4 \mathrm{MPa}$. The failure strain averaged $43 \pm 16 \%$ and $47 \pm 2 \%$ for channels along $\mathrm{x}$ and $\mathrm{y}$ directions, respectively, and $52 \pm 2 \%$ for woodpile. The highest failure stress was displayed by channels along the $\mathrm{x}$ direction $(19 \pm 1.8 \mathrm{MPa})$, while for woodpiles and channels along y direction, respectively, the values were $0.4 \pm 0.2$ and $0.7 \pm 0.1 \mathrm{MPa}$. Altogether these data indicate a remarkable difference in mechanical characteristics between channels and woodpiles and also denote the channel architecture anisotropy (figure 6(B-D)).

Significant differences were observed between PCL channels along x direction and the others in both elastic modulus $(\mathrm{P}<0.05)$ and failure stress $(\mathrm{P}<0.01)$. Starting from the consideration that for the channel-shaped scaffold all layers have fibers oriented in the $\mathrm{x}$ direction, while for the woodpile shaped scaffold half of the layers have fibers oriented in the $\mathrm{x}$ direction, a difference of about the half in mechanical properties was expected: the actual ratio is 2.4 in respect to elastic modulus and 2.8 in respect to failure stress. No significant differences were detected in failure strain between the three groups. Noteworthy, the mechanical features of both fabricated structures are similar to biological values of blood vessels, considering that the elastic modulus of human femoral artery is around 1.2-4 MPa.

\subsection{Comparison of cellularized scaffolds}

Having achieved the fabrication of composite materials, we next moved to the following objective of this study which compared the scaffolds capability to accommodate and be functionalized with human 
cells. APCs were seeded onto the scaffolds and maintained in regular culture medium. Seven days after seeding, we counted the density of cells identified by staining with Dapi (nuclear marker) and Dil (lipophilic membrane dye), while proliferation was assessed by EdU staining, a fluorescent nucleoside which is incorporated into DNA of cells in S-phase (figure 7(A)).

Comparison between different structures indicates that channels electrospun with GPTMS-crosslinked fibres yield the highest cells density (figure 7(B)). Next, we compared the bio-scaffolds with regard to cell viability. Channels electrospun with genipin-crosslinked nanofibres conferred the best structural conditions to preserve viability (figure 7(B)). Importantly, all the scaffolds showed the presence of proliferating cells, thus suggesting they have a good biomimetic structure. Channels electrospun with GPTMS-crosslinked fibres yielded the highest proliferation index (figure 7(B)). In order to select the best performant structure to be tested in vivo, we assigned a score calculated on the rank of each structure in the above biological test ( 1 being the best score in each test). Channels-GPTMS scaffolds yield the best average score (mean 1.66, median 1), followed by woodpiles-GPTMS (mean 2.66, median 2), channels-genipin (mean 2.66, median 3), and woodpiles-genipin (mean 3, median 3). Therefore, the first of these structures was finally implanted perivascularly in a mouse model of limb ischemia. Before moving to the in vivo application, we performed differentiation assays of APCs seeded onto the chosen channel-shaped/GPTMS-crosslinked scaffold. After 14 days culture with inductive media, the expression of typical markers was evaluated by immunocytochemistry. Results confirm previous studies showing that APCs maintain their original phenotype and do not acquire markers of mature vascular cells (figure 7C) [21].

\subsection{In vivo studies}

Endpoints of in vivo studies using channel-shaped/GPTMS-crosslinked scaffolds were the blood flow recovery, collateral formation around the occluded artery and distal angiogenesis in ischemic muscle. Two-way ANOVA indicates an effect of time $(P<0.01)$ and scaffold implantation $(P<0.05)$ on superficial blood flow recovery as assessed by laser Doppler flowmetry (Figure 8(A)). However, there was no difference between scaffolds alone or scaffolds seeded APCs. In contrast, a significant improvement was denoted in mice given cell-engineered scaffolds with regard to adductor muscle blood flow measured by fluorescent microspheres $(1.3 \pm 0.1$ vs. $1.0 \pm 0.1$ and $0.7 \pm 0.1 \mathrm{ml} / \mathrm{min} / \mathrm{gm}$ of tissue in mice given scaffold alone or vehicle, respectively, figure 8(B)). With regard to neovascularization of ischemic adductor muscles, arteriole density, but not capillary density, was increased by scaffolds with or without cells $(P<0.01$ vs. vehicle for both comparisons, figure $8(C \& D))$. Additionally, there was a remarkable increase in arterial collaterals in the space between the scaffold and femoral artery, with this effect being significantly greater in mice implanted with APCengineered scaffolds (figure 8(E)).

The scaffold was still visible at 3 weeks from implantation but appeared fragmented, which suggests initial degradation. Furthermore, a remarkable recruitment of F4/80 monocytes was observed, with no difference in cells infiltration between groups that received the scaffold alone or APC-engineered 
scaffolds. Altogether, in vivo data indicate that the scaffold itself has the ability to promote an improvement in proximal and distal vascularization and that APC-engineering additively implements this capacity at the proximal perivascular site. Immunohistochemistry analyses excluded the presence of APCs at 21 days from implantation, thus suggesting that paracrine mechanisms are mainly responsible for the improvement of reparative neovascularization.

\section{Discussion}

In the present study, we developed new synthetic biomaterials consisting of 3D scaffolds containing human stem cells for therapeutic use in a model of peripheral ischemia. Results indicate that perivascular provision of human APCs within novel composite materials results in remarkable proximal collateral formation, distal angiogenesis and acceleration of blood flow recovery.

New generations of cellularized biomaterials are being developed for use as 3D extracellular microenvironments to mimic the characteristics of natural ECM and create the temporal and spatial complexity instrumental to tissue- and organ-specific morphogenesis. Synthetic biodegradable polylactones such as PCL are now commonly employed in biomedical devices because of their excellent biocompatibility, biodegradability, thermal plasticity and suitable mechanical properties. We exploited PCL characteristics to fabricate 3D structures capable of hosting human stem cells according to a defined geometry for the purpose of promoting a well aligned collateralization upon in vivo implantation. We used a novel micro-positioning system equipped with a pressure-controlled syringe to fabricate high-resolution structures with the shape of channels and woodpiles, which are ideal to guide tissue regeneration along the principal directions of porosity. Scaffolds were then enmeshed with a nanofibre network to increase adhesive properties and cross-linked with genipin or GPTMS, which confer the internal gelatin core with elasticity and durability.

Results of mechanical tests indicate the fabrication method's capability to control features such as anisotropy, stiffness and elasticity and to reproduce the natural physical properties of living tissues. The topology of fabricated scaffold allows to obtain, when it is shaped in tubular structure for in vivo test, different mechanical properties in the circumferential and longitudinal directions, mimicking the in vivo biomechanical behaviour of natural vascular structure.

Importantly, in vitro assays demonstrate the capability of engineered structures to support stem cell adhesion, viability and proliferation. In this respect, channels structures were superior to woodpiles. Furthermore, structures fabricated with the crosslinking agent GPTMS over-performed those using genipin. The former agent has been previously used for anchoring ECM proteins on microscale spots and long-term culturing of MSCs. Here, we show for the first time the usefulness of GPTMS in aiding the biocompatibility of PCL scaffolds.

After arterial occlusion, pre-existing vessels enlarge and remodel through an arteriogenic process that is triggered by shear stress and involves soluble factors, cell proliferation, and ECM 
rearrangement. We found that the arteriogenic process is enhanced by perivascular implantation of cellularized bioscaffolds and, to a lesser extent, by the scaffolds themselves. This represents a significant improvement over conventional intramuscular cell therapy, which disperses cells and therapeutic mediators in an unpredictable manner. In addition, perivascular delivery of scaffolds enhanced the tributary microvascular bed in hindlimb muscles, as documented by increased counts of small arterioles in the ischemic adductor. The absence of human APCs at the implantation site indicates that the observed therapeutic benefit derives from stimulation of endogenous mechanisms of vascular repair. This result is reassuring on a safety standpoint, as prolonged persistence of proangiogenic cells may cause undesired effects and potentially also increase the risk of cancer.

In summary, the described methodology using composite biomaterials functionalized with APCs may offer potential as a treatment for peripheral vascular disease, whereby administration of the cell-engineered material within the vicinity of diseased vessels could promote revascularization and re-establish blood flow to the ischemic limb. The approach is particularly attractive for use in patients in whom interventional revascularization is not amenable because of multiple or distal obstructions. Moreover, perivascular cell therapy might be used as an adjuvant treatment in conjunction with or preparation to operative revascularization.

\section{Acknowledgments}

This work was supported with funds from the British Heart Foundation (Studentship grant and Pump priming grant from the Center of Vascular Regenerative Medicine) and Medical Research Council to Paolo Madeddu.

\section{References}

[1] Hiatt WR. Medical treatment of peripheral arterial disease and claudication. N Engl J Med. 2001;344:1608-21.

[2] Faglia E, Clerici G, Clerissi J, Gabrielli L, Losa S, Mantero M, et al. Early and five-year amputation and survival rate of diabetic patients with critical limb ischemia: data of a cohort study of 564 patients. Eur J Vasc Endovasc Surg. 2006;32:484-90.

[3] Faglia E, Clerici G, Losa S, Tavano D, Caminiti M, Miramonti M, et al. Limb revascularization feasibility in diabetic patients with critical limb ischemia: results from a cohort of 344 consecutive unselected diabetic patients evaluated in 2009. Diabetes Res Clin Pract. 2012;95:364-71.

[4] Blum A, Balkan W, Hare JM. Advances in cell-based therapy for peripheral vascular disease. Atherosclerosis. 2012;223:269-77. 
[5] Tongers J, Roncalli JG, Losordo DW. Therapeutic agniogenesis for critical limb ischemia: microvascular therapies coming of age. Circulation. 2008;118:9-16.

[6] Matoba S, Tatsumi T, Murohara T, Imaizumi T, Katsuda Y, Ito M, et al. Long-term clinical outcome after intramuscular implantation of bone marrow mononuclear cells (Therapeutic Angiogenesis by Cell Transplantation [TACT] trial) in patients with chronic limb ischemia. Am Heart J. 2008;156:1010-8.

[7] Burdon TJ, Paul A, Noiseux N, Prakash S, Shum-Tim D. Bone marrow stem cell derived paracrine factors for regenerative medicine: current perspectives and therapeutic potential. Bone Marrow Res. 2011;2011:207326.

[8] Ruiz-Salmeron R, de la Cuesta-Diaz A, Constantino-Bermejo M, Perez-Camacho I, MarcosSanchez F, Hmadcha A, et al. Angiographic demonstration of neoangiogenesis after intra-arterial infusion of autologous bone marrow mononuclear cells in diabetic patients with critical limb ischemia. Cell Transplant. 2011;20:1629-39.

[9] Powell RJ. Update on clinical trials evaluating the effect of biologic therapy in patients with critical limb ischemia. J Vasc Surg. 2012;56:264-6.

[10] Weber C, Pohl S, Poertner R, Pino-Grace P, Freimark D, Wallrapp C, et al. Production process for stem cell based therapeutic implants: expansion of the production cell line and cultivation of encapsulated cells. Adv Biochem Eng Biotechnol. 2010;123:143-62.

[11] Goren A, Dahan N, Goren E, Baruch L, Machluf M. Encapsulated human mesenchymal stem cells: a unique hypoimmunogenic platform for long-term cellular therapy. FASEB J. 2010;24:22-31.

[12] Montanucci P, Pennoni I, Pescara T, Blasi P, Bistoni G, Basta G, et al. The functional performance of microencapsulated human pancreatic islet-derived precursor cells. Biomaterials. 2011;32:9254-62.

[13] Hernandez RM, Orive G, Murua A, Pedraz JL. Microcapsules and microcarriers for in situ cell delivery. Adv Drug Deliv Rev. 2010;62:711-30.

[14] Katare R, Riu F, Rowlinson J, Lewis A, Holden R, Meloni M, et al. Perivascular delivery of encapsulated mesenchymal stem cells improves postischemic angiogenesis via paracrine activation of VEGF-A. Arterioscler Thromb Vasc Biol. 2013;33:1872-80.

[15] Martins-Green. Dynamics of cell-ECM interaction. 2nd ed ed: Academic Press; 2000.

[16] Anuradha Subramanian UM, Swaminathan Sethuraman. 2012.

[17] Tirella A, Vozzi F, Vozzi G, Ahluwalia A. PAM2 (piston assisted microsyringe): a new rapid prototyping technique for biofabrication of cell incorporated scaffolds. Tissue Eng Part C Methods. 2011;17:229-37.

[18] Yeo M, Lee H, Kim G. Three-dimensional hierarchical composite scaffolds consisting of polycaprolactone, $\beta$-tricalcium phosphate, and collagen nanofibres: fabrication, physical properties, and in vitro cell activity for bone tissue regeneration. Biomacromolecules. 2011;12:502-10. 
[19] Vono R, Spinetti G, Gubernator M, Madeddu P. What's new in regenerative medicine: split up of the mesenchymal stem cell family promises new hope for cardiovascular repair. J Cardiovasc Transl Res. 2012;5:689-99.

[20] Spencer HL, Slater SC, Rowlinson J, Morgan T, Culliford LA, Guttridge M, et al. A journey from basic stem cell discovery to clinical application: the case of adventitial progenitor cells. Regen Med. 2015;10:39-47.

[21] Campagnolo P, Cesselli D, Al Haj Zen A, Beltrami AP, Krankel N, Katare R, et al. Human adult vena saphena contains perivascular progenitor cells endowed with clonogenic and proangiogenic potential. Circulation. 2010;121:1735-45.

[22] Gubernator M, Slater SC, Spencer HL, Spiteri I, Sottoriva A, Riu F, et al. Epigenetic profile of human adventitial progenitor cells correlates with therapeutic outcomes in a mouse model of limb ischemia. Arterioscler Thromb Vasc Biol. 2015;35:675-88.

[23] Katare R, Riu F, Mitchell K, Gubernator M, Campagnolo P, Cui Y, et al. Transplantation of human pericyte progenitor cells improves the repair of infarcted heart through activation of an angiogenic program involving micro-RNA-132. Circ Res. 2011;109:894-906.

[24] Avolio E, Meloni M, Spencer HL, Riu F, Katare R, Mangialardi G, et al. Combined intramyocardial delivery of human pericytes and cardiac stem cells additively improves the healing of mouse infarcted hearts through stimulation of vascular and muscular repair. Circ Res. 2015;116:e8194.

[25] Tirella A, De Maria C, Criscenti G, al. e. The $\mathrm{PAM}^{2}$ system: a multilevel approach for fabrication of complex threedimensional microstructures. Rapid Prototyp J. 2012;18:299-307-9.

[26] Cei D, Malena A, de Maria C, Loro E, Sandri F, Del Moro G, et al. In vitro development of engineered muscle using a scaffold based on the pressure-activated microsyringe (PAM) technique. $\mathrm{J}$ Tissue Eng Regen Med. 2014; doi: 10.1002/term.1894.

[27] Tonda-Turo C, Cipriani E, Gnavi S, Chiono V, Mattu C, Gentile P, et al. Crosslinked gelatin nanofibres: preparation, characterisation and in vitro studies using glial-like cells. Mater Sci Eng C Mater Biol Appl. 2013;33:2723-35.

[28] Panzavolta S, Gioffre M, Focarete ML, Gualandi C, Foroni L, Bigi A. Electrospun gelatin nanofibers: optimization of genipin cross-linking to preserve fiber morphology after exposure to water. Acta Biomater. 2011;7:1702-9.

[29] Katare R, Stroemer P, Hicks C, Stevanato L, Patel S, Corteling R, et al. Clinical-grade human neural stem cells promote reparative neovascularization in mouse models of hindlimb ischemia. Arterioscler Thromb Vasc Biol. 2014;34:408-18.

[30] Powell HM, Boyce ST. Fiber density of electrospun gelatin scaffolds regulates morphogenesis of dermal-epidermal skin substitutes. J Biomed Mater Res A. 2008;84:1078-86. 
[31] Min BM, Lee G, Kim SH, Nam YS, Lee TS, Park WH. Electrospinning of silk fibroin nanofibers and its effect on the adhesion and spreading of normal human keratinocytes and fibroblasts in vitro. Biomaterials. 2004;25:1289-97.

[32] Christopherson GT, Song H, Mao HQ. The influence of fiber diameter of electrospun substrates on neural stem cell differentiation and proliferation. Biomaterials. 2009;30:556-64.

[33] Lu LX, Wang YY, Mao X, Xiao ZD, Huang NP. The effects of PHBV electrospun fibers with different diameters and orientations on growth behavior of bone-marrow-derived mesenchymal stem cells. Biomed Mater. 2012;7:015002. 

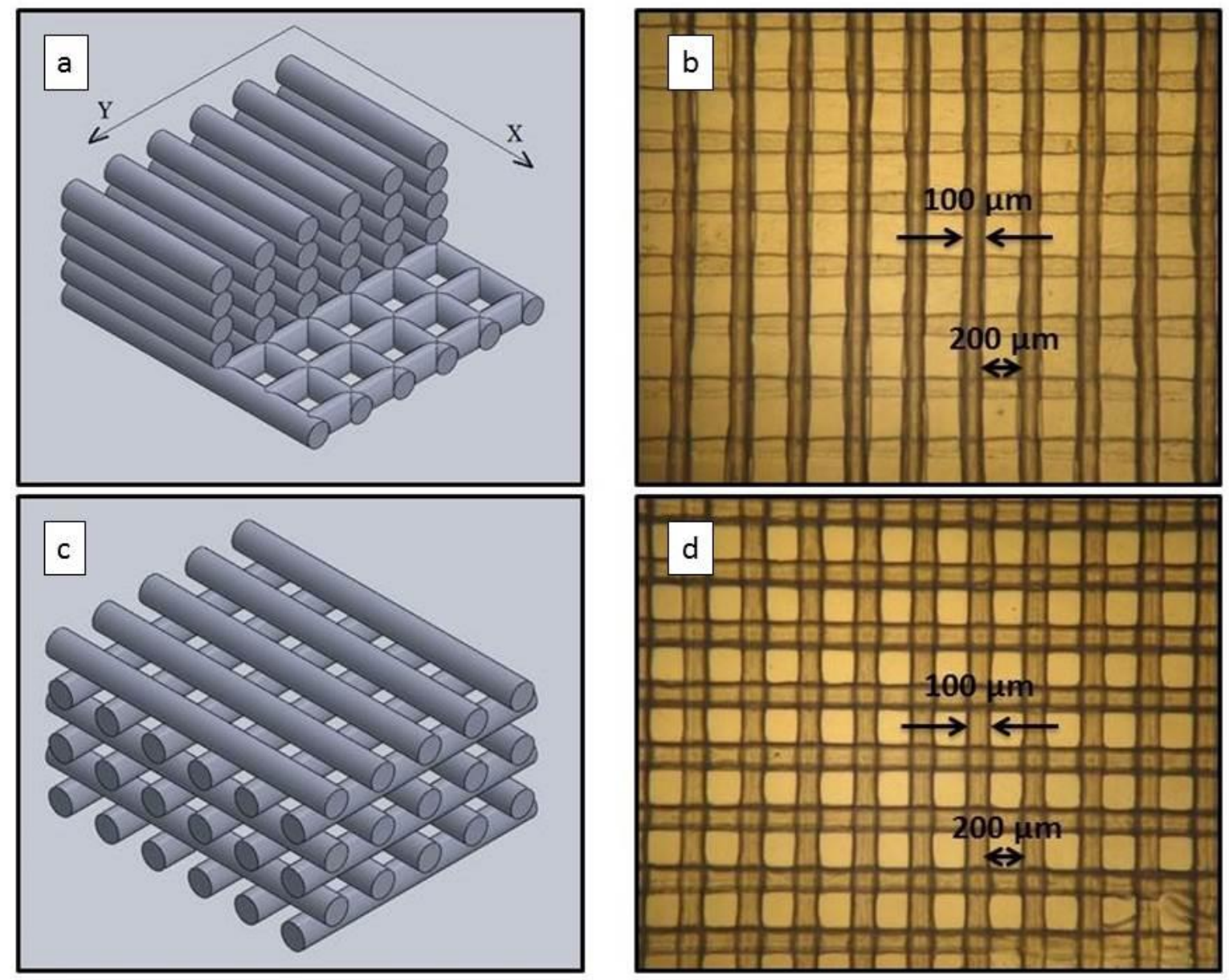

Figure 1: (a) 3D render showing the $x$ and $y$ axis of fabricated channel structures, and (b) microphotograph (captured by optical microscope AX 70 Olympus, Tokyo, Japan) of channel structure made by PAM ${ }^{2}$; (c) render and (d) microphotograph of woodpiles made by PAM $^{2}$. 


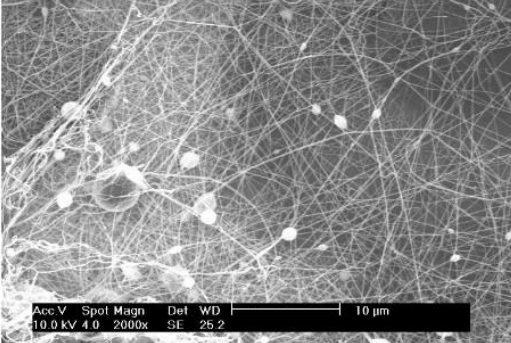

(A)

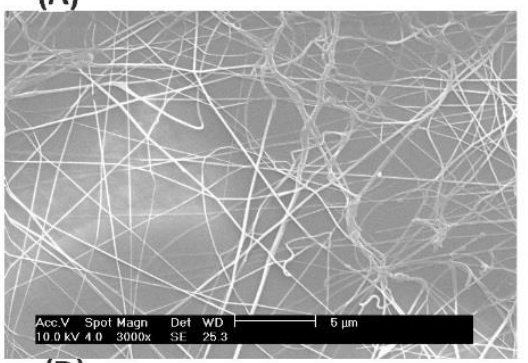

(D)

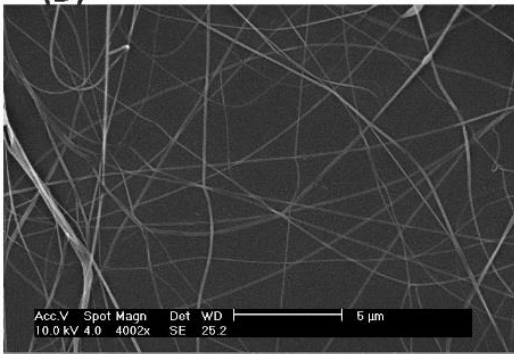

(G)

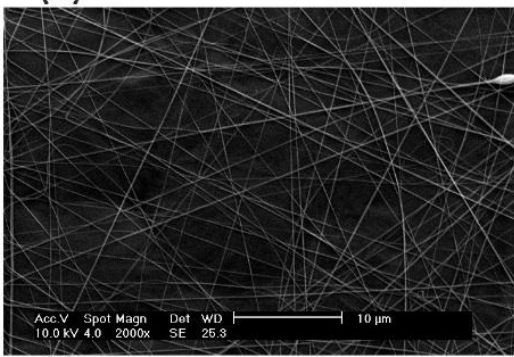

(J)

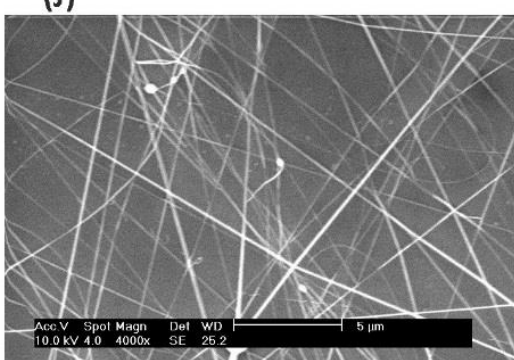

(M)

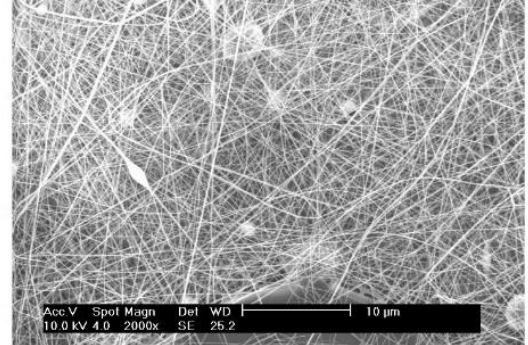

(B)

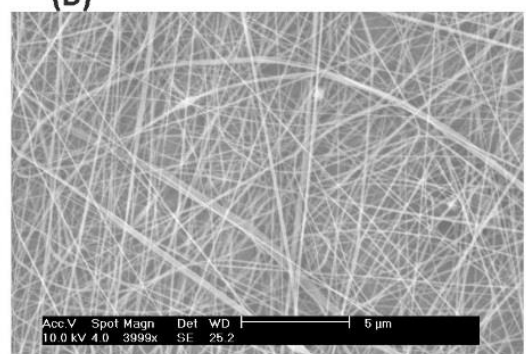

(E)

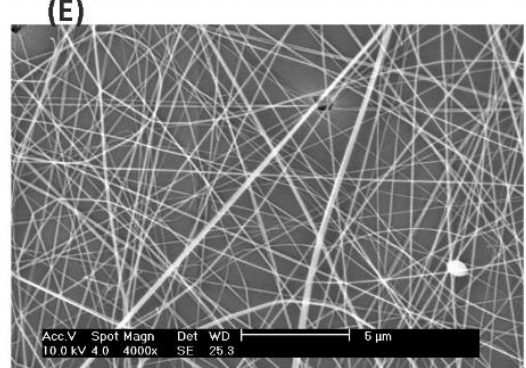

(H)

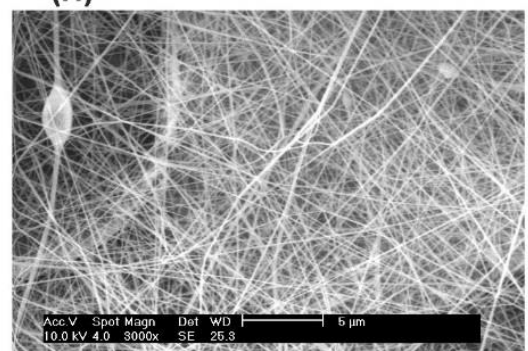

(K)

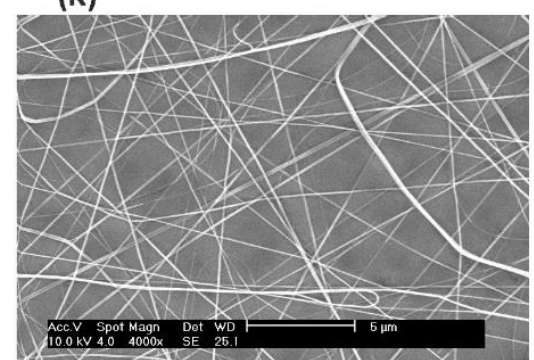

(N)

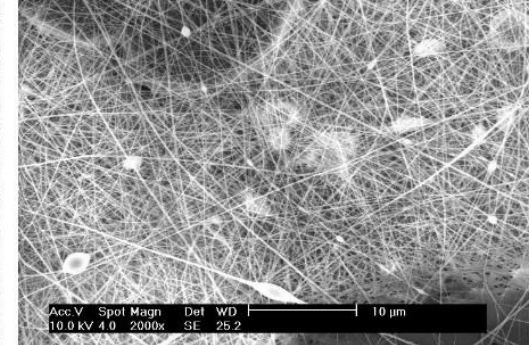

(C)

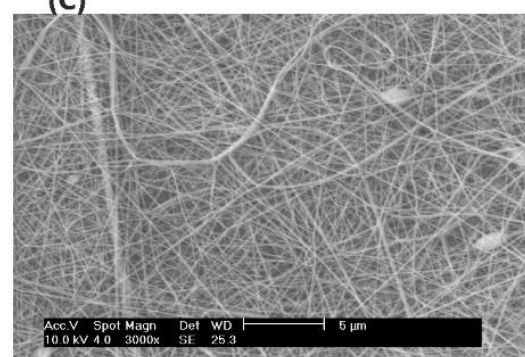

(F)

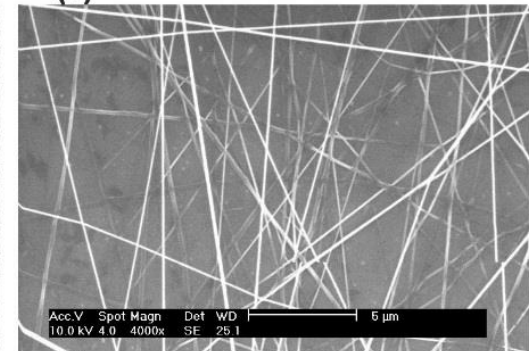

(I)

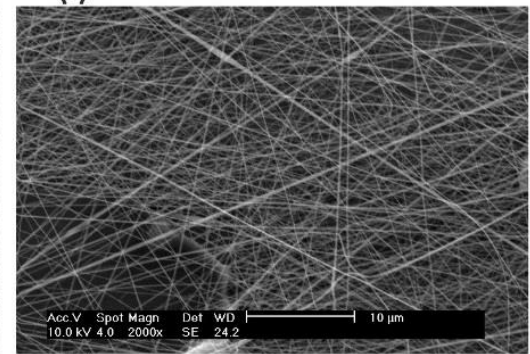

(L)

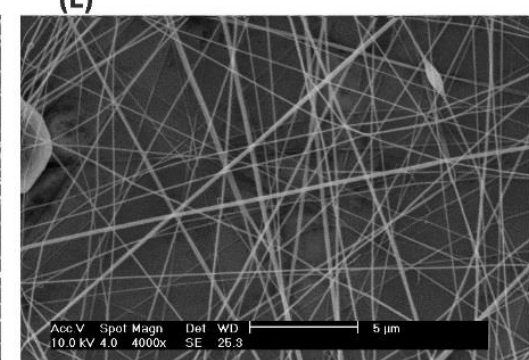

(O)

Figure 2: Scanning electron microscopy microphotographs of nanofibres obtained varying the process parameters (d: distance; v: voltage; f: flow rate): (A) $15 \mathrm{~cm}, 40 \mathrm{kV}, 1 \mathrm{ml} / \mathrm{hour}$; (B) $15 \mathrm{~cm}, 40 \mathrm{kV}, 2 \mathrm{ml} / \mathrm{hour}$; (C) $15 \mathrm{~cm}, 40 \mathrm{kV}, 3 \mathrm{ml} /$ hour; (D) $15 \mathrm{~cm}, 50 \mathrm{kV}, 1 \mathrm{ml} /$ hour; (E) $15 \mathrm{~cm}, 50 \mathrm{kV}, 2 \mathrm{ml} /$ hour; (F) $15 \mathrm{~cm}, 50 \mathrm{kV}, 3$ ml/hour; (G) $20 \mathrm{~cm}, 40 \mathrm{kV}, 1 \mathrm{ml} /$ hour; (H) $20 \mathrm{~cm}, 40 \mathrm{kV}, 2 \mathrm{ml} /$ hour; (I) $20 \mathrm{~cm}, 40 \mathrm{kV}, 3 \mathrm{ml} /$ hour; (J) $20 \mathrm{~cm}, 50$ $\mathrm{kV}, 1 \mathrm{ml} /$ hour; (K) $20 \mathrm{~cm}, 50 \mathrm{kV}, 2 \mathrm{ml} / \mathrm{hour}$; (L) $20 \mathrm{~cm}, 50 \mathrm{kV}, 3 \mathrm{ml} /$ hour; (M) $30 \mathrm{~cm}, 50 \mathrm{kV}, 1 \mathrm{ml} /$ hour; (N) 30 $\mathrm{cm}, 50 \mathrm{kV}, 2 \mathrm{ml} /$ hour; (O) $30 \mathrm{~cm}, 50 \mathrm{kV}, 3 \mathrm{ml} /$ hour. 

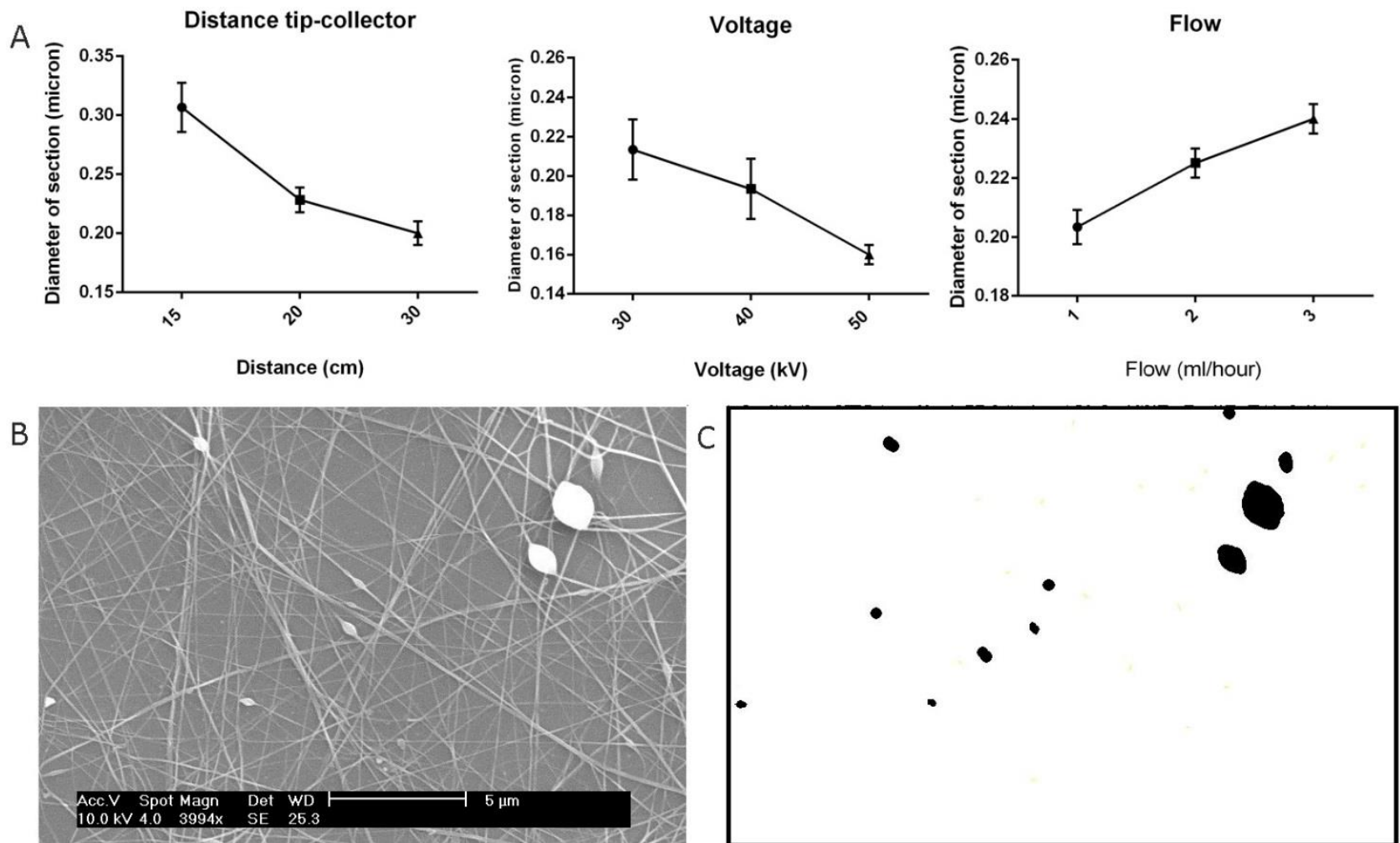

Figure 3: (A) Linear graphs showing the dependency of diameter of nanofibre section on electrospinning parameters: distance, voltage and flow rate. Values expressed as mean \pm standard error. Analysis of nanofibres defects; (B) SEM micrograph of nanofibres; fibres show irregularity due to electrospinning process. (C) Defects are isolated by image processing and quantified in terms of number of pixel of defects. 
A

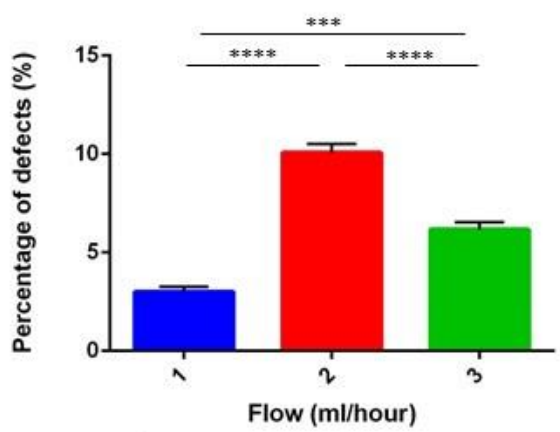

D

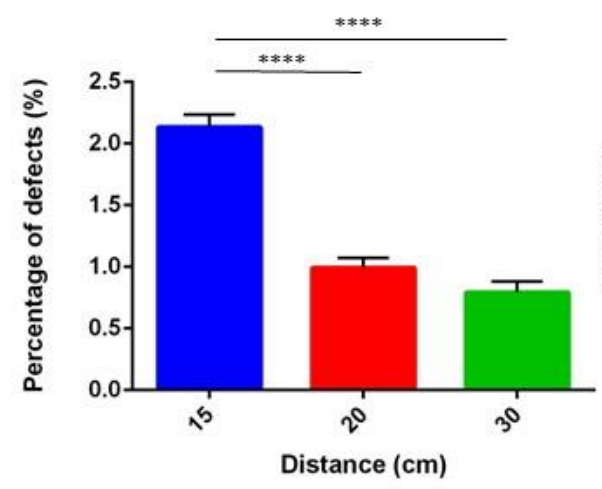

B

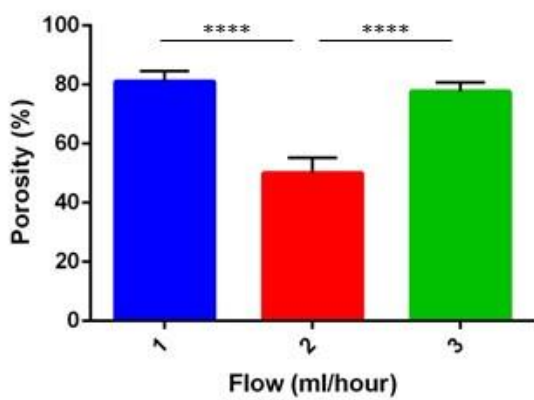

E

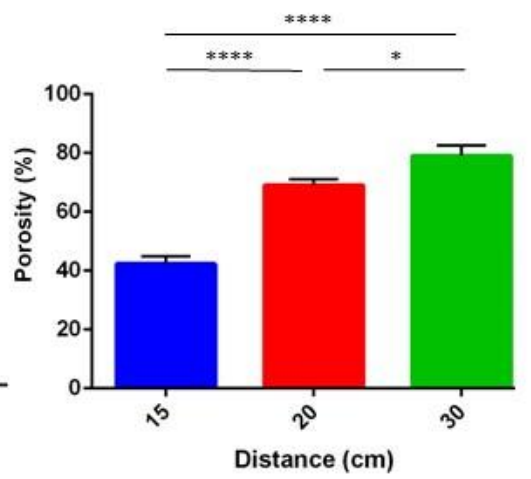

C

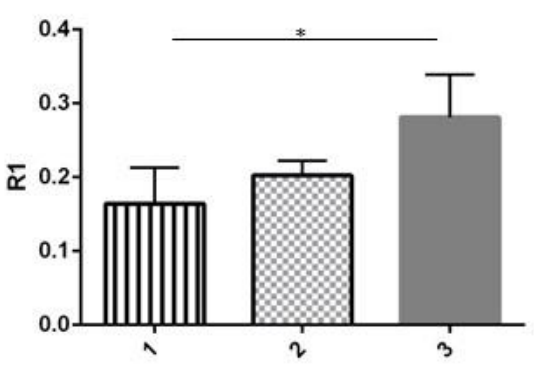

Flow (ml/hour)

F

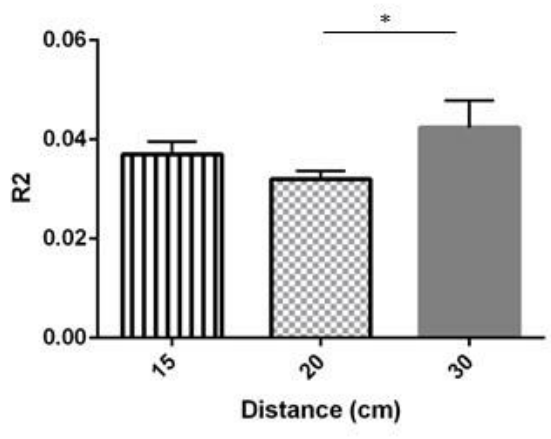

Figure 4: Bar graphs showing percentage of nanofibre defects (A) and porosity (B) in relation to flow rate. (C) Bar graph of variable R1 assessed by calculating the ratio between defects and fibres for different distance values. Bar graphs showing percentage of nanofibre defects (D) and porosity (E) in relation to tip-collector distance. (F) Bar graph of variable R2 assessed by calculating the ratio between defects and fibres for different flow rate. $* \mathrm{P}<0.05 ; * * \mathrm{P}<0.01 ; * * * \mathrm{P}<0.001$. $* * * * \mathrm{P}<0.0001$. Values expressed as mean \pm standard error. 

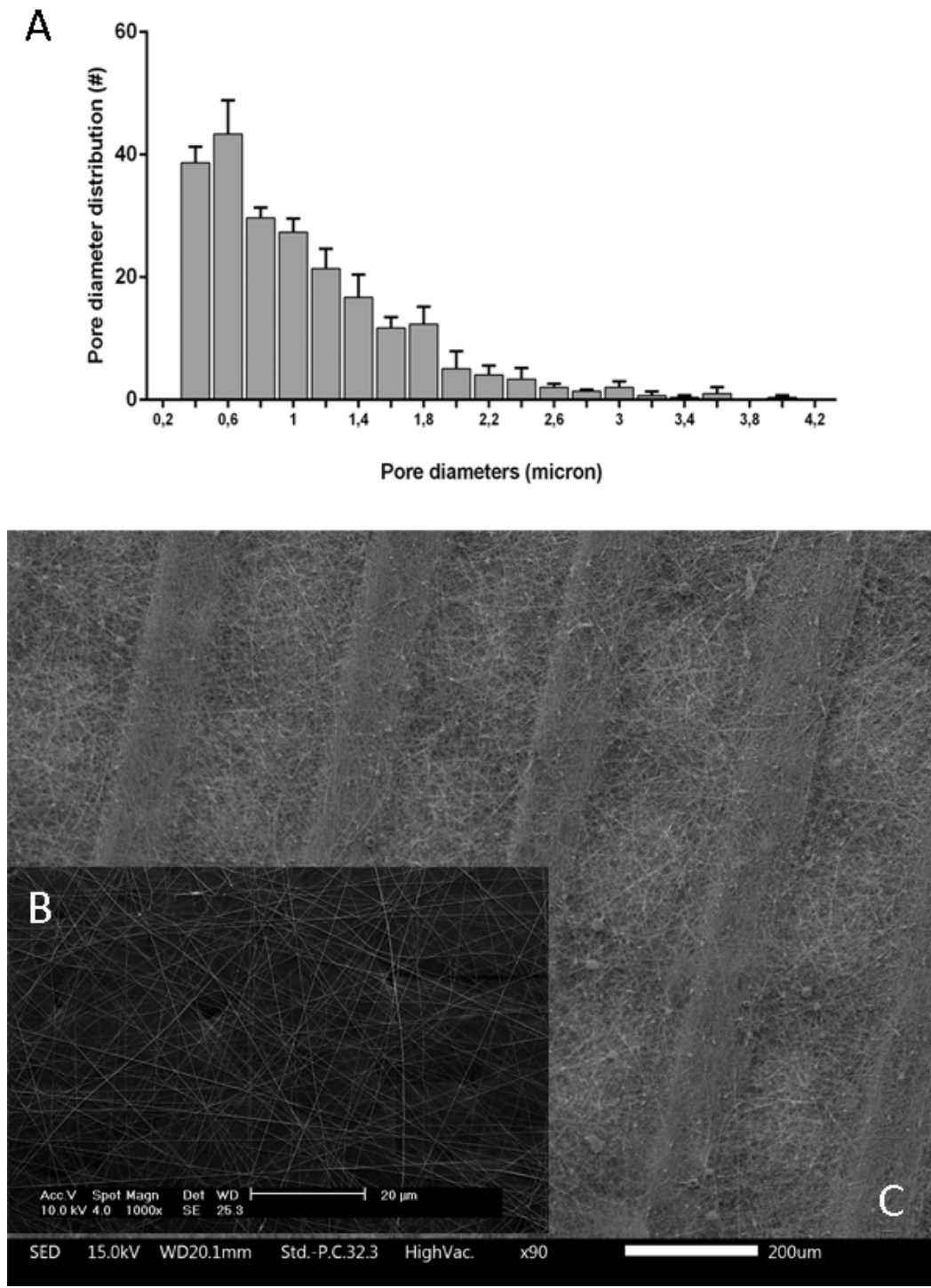

Figure 5: (A) Bar graph of pore diameter distribution of the nanofibre mat chosen after the tuning process; (B) Scanning electron microscopy microphotograph of nanofibres mat obtained after tuning of working conditions; (C) Scanning electron microscopy microphotographs showing structures obtained by combination of PAM ${ }^{2}$ and electrospinning. 
A
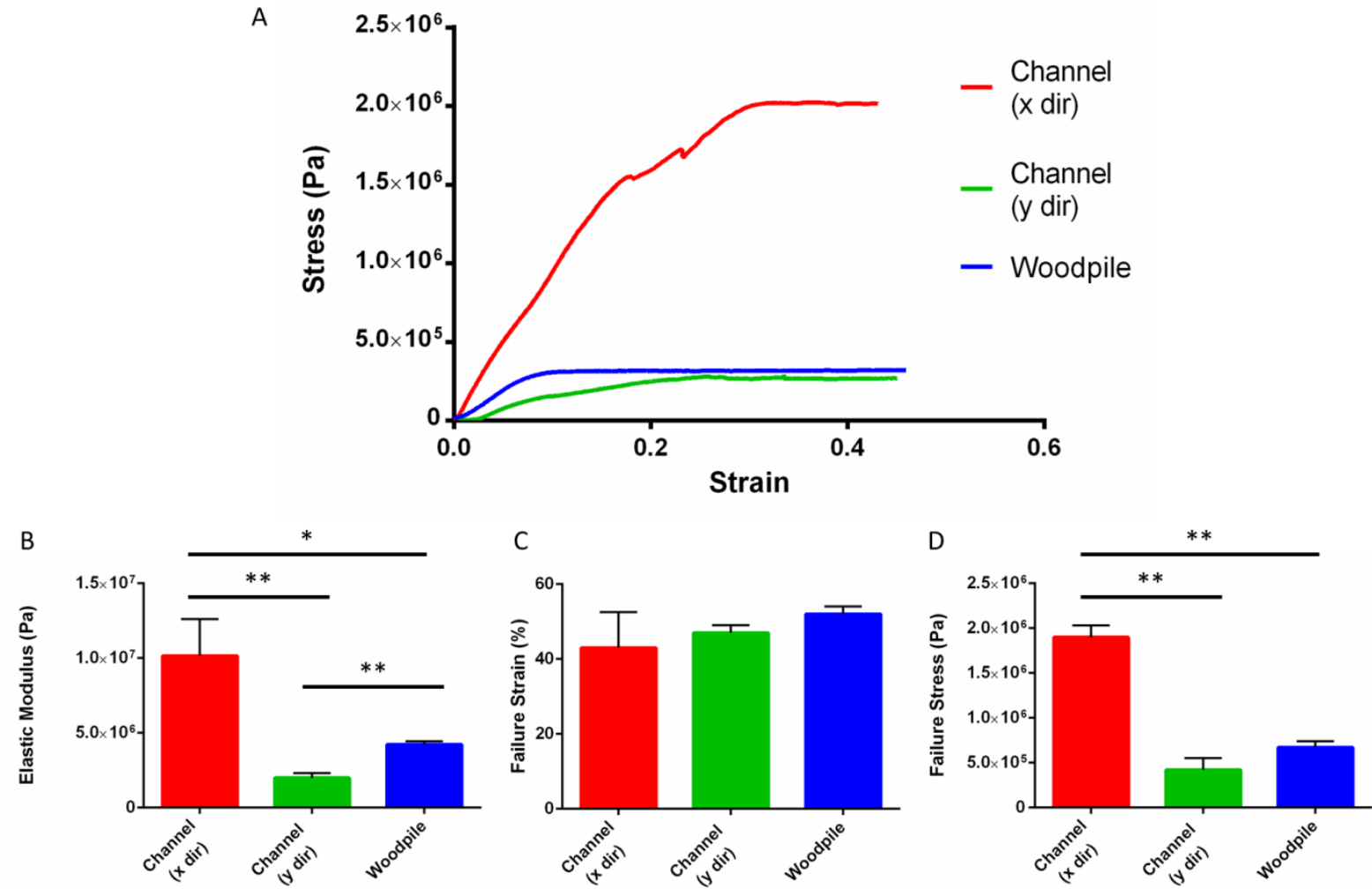

Figure 6: (A) Stress-strain curve of PCL structures. Bar graphs summarize results of mechanical tests on PCL structures: (B) Elastic Modulus; (C) Failure Strain; (D) Failure Stress. $* \mathrm{P}<0.05$; $* * \mathrm{P}<0.01 ; * * * \mathrm{P}<0.001$. $* * * * \mathrm{P}<0.0001$. Values expressed as mean \pm standard error. 


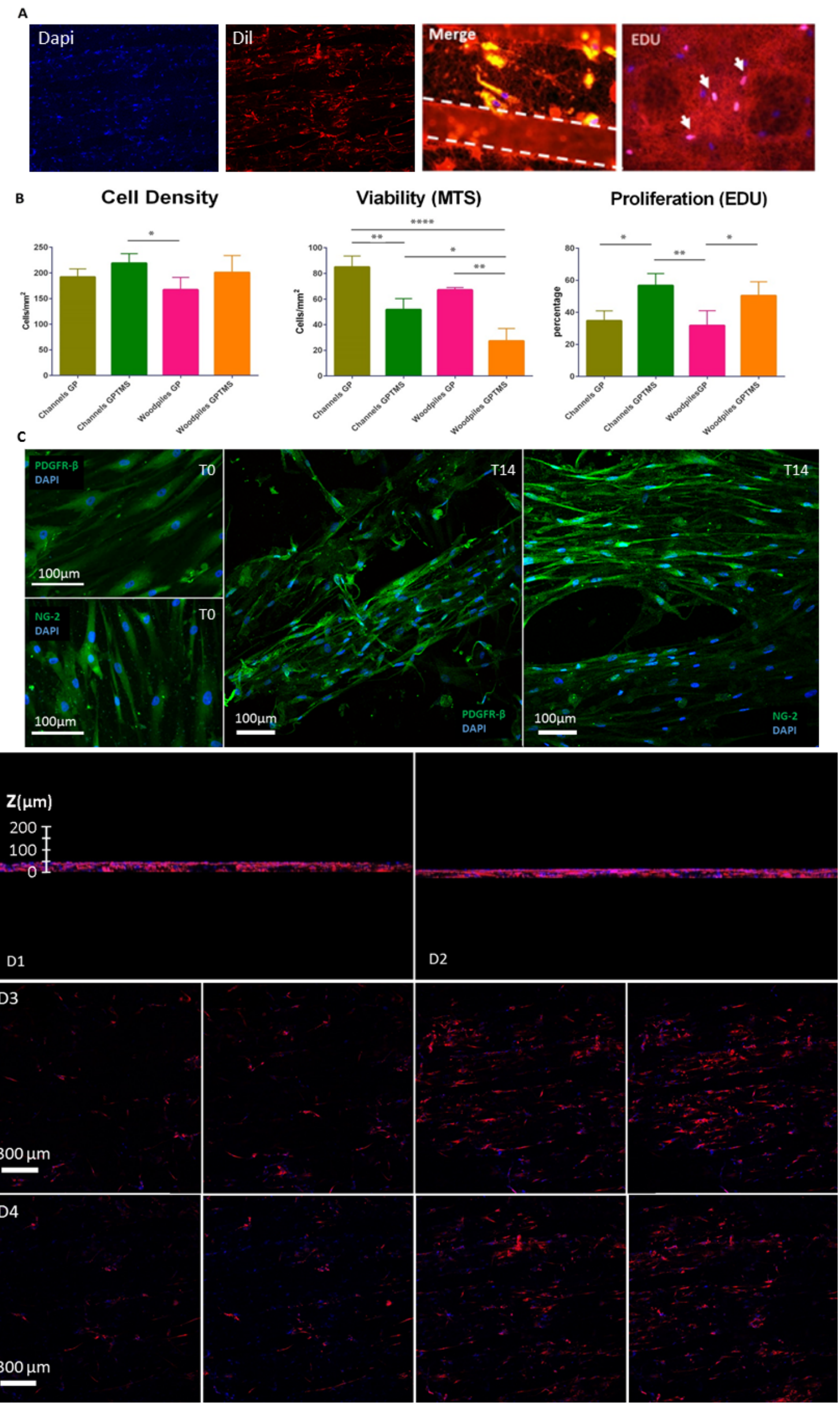


Figure 7: In vitro cellularization of scaffolds. (A) Representative fluorescent microscopy images of scaffolds seeded with APCs. Nuclei are stained blue with DAPI and cell bodies are labelled with Dil. Magnification image shows cells in the channels delimited by the wall (dotted line). Proliferating cells are stained pink by EdU. (B) Bar graphs summarize results of in vitro assays on APCs seeded on hybrid scaffolds. Gelatin nanofibres were crosslinked with genipin and GPTMS. (C) Pictures show the maintaining of the APCs markers expression, PDGFR- $\beta$ and NG-2, from T0, the day in which cells were seeded on scaffold, and T14, the day in which cells were analysed by cytochemistry. (D1-4) APCs distribution in channel and woodpile scaffolds; Z stack reconstruction of confocal microscopy images from channel scaffolds (D1) and woodpile scaffolds (D2). Defining $\mathrm{z}=0$ as the bottom of the scaffold, moving from left to right, four sections of cells grown on channel (D3) or woodpile scaffold (D4) are illustrated at different height at10 $\mu \mathrm{m} ; \mathrm{z}=20 \mu \mathrm{m}, \mathrm{z}=30 \mu \mathrm{m}, \mathrm{z}=40 \mu \mathrm{m}$. $* \mathrm{P}<0.05 ; * * \mathrm{P}<0.01 ; * * * \mathrm{P}<0.001$. **** $\mathrm{P}<0.0001$.

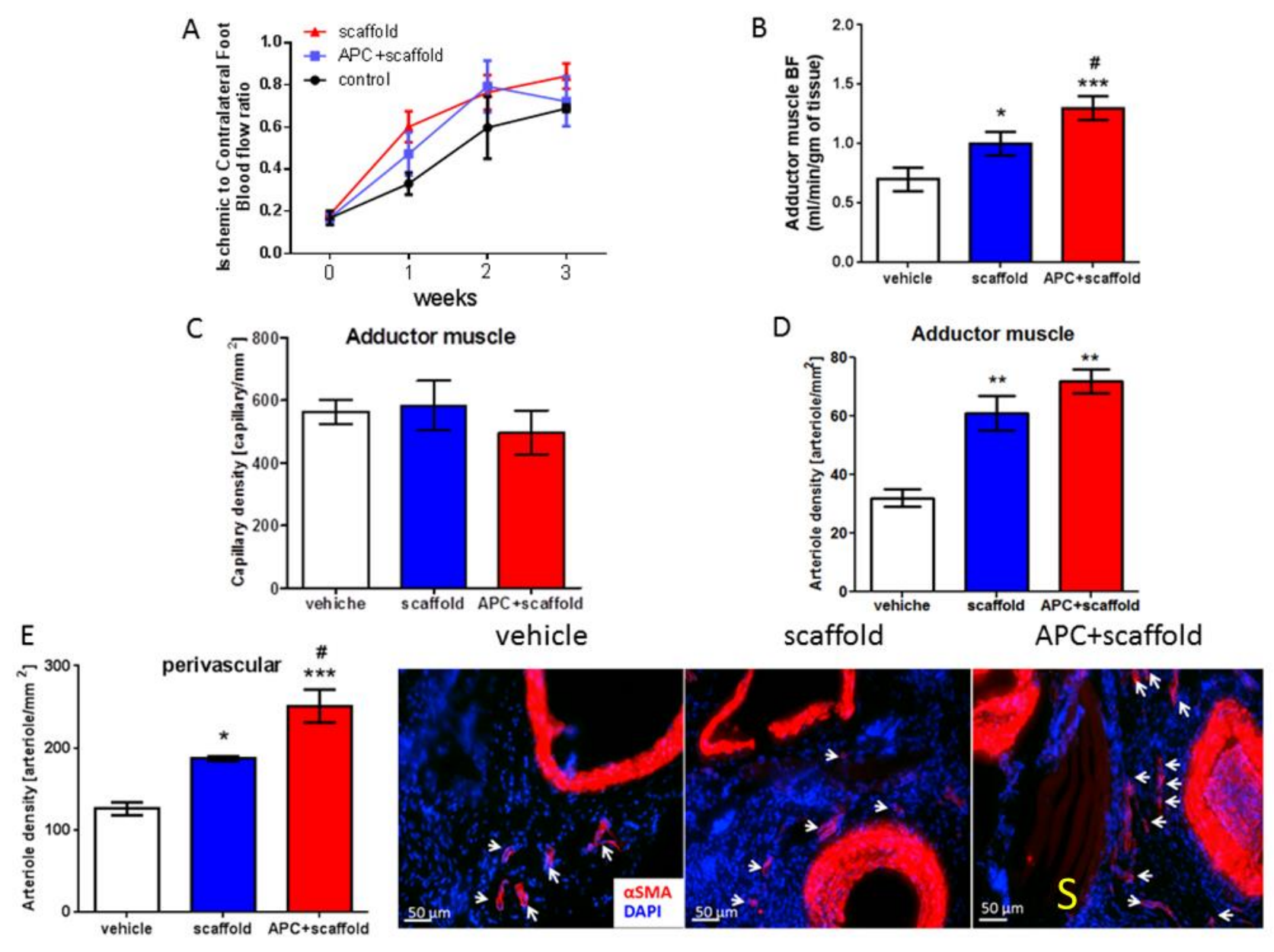

Figure 8: Perivascular implantation of scaffolds. (A) Blood flow recovery assessed by laser Doppler flowmetry (B) and fluorescent microspheres in adductor muscles, (C) Capillary and (D) arteriole density in limb muscles, (E) Arteriole density in the implantation site; bar graph and immunohistochemistry of vascularization around the femoral artery. Nuclei stained blue by Dapi and vascular smooth muscle cells stained red by alpha-smooth muscle actin. Arrows indicate arterioles. S indicates the scaffold. $* \mathrm{P}<0.05$ and $* * * \mathrm{P}<0.001$ vs. vehicle. \#P<0.01 vs. scaffold alone. 